\title{
Dörner, Andreas/Vogt, Ludgera (Hrsg.): Mediale Störungen. Krisenkommunikation in Sondersendungen des deutschen Fernsehens.
}

\author{
Wiesbaden: Springer VS 2020. 362 Seiten. Preis: $€ 44,99$ (e-book)
}

\section{Sebastian Köhler}

Angenommen: 2. September 2021 / Online publiziert: 28. September 2021

(C) Der/die Autor(en) 2021

Der Band Mediale Störungen erschien, bevor die globale Corona-Krise seit März 2020 bisher beispiellose gesellschaftliche „Störungen“ mit sich brachte. Dennoch kann diese Mega-Krise als Indiz dafür gelesen werden, dass die Thematik des Sammelbandes beträchtliche gesellschaftliche Relevanz und Aktualität aufweist. In einer offenbar von allgemeinen und besonderen Krisen geprägten Gesellschaft hängen soziale und mediale „Störungen“ womöglich zunehmend zusammen. Die DFG förderte ein entsprechendes Projekt mit Fokus auf Fernsehsondersendungen, dessen Forschungsergebnisse im vorliegenden Band dargelegt werden. Vogt und Dörner zufolge liegen zu diesem Themenbereich bisher kaum systematische Erkenntnisse vor.

Die HerausgeberInnen sahen bereits ,,vor Corona“ die Gefahr möglicher Inflationierungen von Sondersendungen. Sie konzentrierten sich mit ihren Studien sehr deutlich auf Sondersendungen öffentlich-rechtlicher Anstalten, vor allem auf „ARD Brennpunkt“ und „ZDF spezial“" der Jahre 2015 und 2016. Die AutorInnen des Bandes gehen davon aus, dass „Brennpunkt“ und „Spezial“ zu den wichtigsten Institutionen der deutschen Medienkultur zählen. Sie sprechen gerade den öffentlichrechtlichen Sondersendungen eine ,große Prominenz“ zu, die sie nicht zuletzt mit „beachtlichen Reichweiten von teilweise bis zu 10 Mio.“ Zuschauenden begründen. Das rechtfertige es, dieses „Genre“ erstmals einer systematischen Analyse zu unterziehen. Bemerkenswert, dass die Herausgebenden von vornherein davon ausgehen, dass die Informationskompetenz des öffentlich-rechtlichen Fernsehens in solchen Sondersendungen ,,besonders deutlich zum Tragen“ kommen soll.

Prof. Dr. Sebastian Köhler ( $\square)$

HMKW Berlin - Hochschule für Medien, Kommunikation und Wirtschaft,

Ackerstraße 76, 13355 Berlin, Deutschland

E-Mail: s.koehler@hmkw.de 
Zentrale Frage des Bandes ist, wie die Programminstitution der Sondersendung systematisch Störungen, Entstörungen und die Re-Etablierung von Ordnung ,definiert, inszeniert und rituell verarbeitet“ (S. $21 \mathrm{f}$.). Es werden die störungstheoretischen Dimensionen Intensität, Topizität, Temporalität, Performativität und Medialität untersucht. Ein wichtiges, relevantes und klar formuliertes Thema des Bandes ist dabei der öffentliche-rechtliche Umgang mit dem Dilemma, abwägen zu müssen zwischen der Vermeidung (zumindest des Eindruckes) einer tatsächlichen Inflationierung von Sondersendungen und dem hohen Legitimationswert solcher Formate gerade für die längst nicht mehr unumstrittenen öffentlich-rechtlichen Rundfunkanstalten. Wichtig mit Blick auf (besser) gelingende gesellschaftliche Kommunikation sind auch der Intensitäts-Aspekt, inwiefern Rückmeldungen von Nutzenden ,für die verschiedenen Akteure eine Rolle spielen“ (S. 23), und der Medialitäts-Aspekt, inwieweit neben Normalisierungen nicht zuletzt Lernprozesse in Richtung sozialer Veränderungen ermöglicht würden.

Was die theoretische Fundierung angeht, bewegen sich die AutorInnen im Kontext störungstheoretischer Modellierungen. Vor diesem Hintergrund benennen sie vier Funktionen der Sondersendungs-Formate thesenhaft: 1) Als „Alarmanlagen“ verwiesen die Formate zumindest diffus auf „Handlungsbedarf“; 2) Die Formate zeigten nicht nur, dass etwas Wichtiges passiert sei, sondern auch, was genau passiert sei, worin etwaige Ursachen lägen, „wie das Ganze zu verstehen und einzuordnen“ sei; 3) die Zuschauenden würden in emotionale Entstörungsrituale eingebunden; und 4) zugleich auch in solche Entstörungsrituale, die in Richtung möglicher Problemlösungen gingen (was m.E. Anknüpfungspunkte in Richtung des „Konstruktiven Journalismus“ bieten könnte). Störungen gelten hier im Sinne systemtheoretischer Modellierungen als Irritationen, die für soziale Systeme ,überlebensnotwendig“ seien (S. 9). Auf systemischer Ebene geht es daher darum, dass solche Störungen als Unterbrechungen einer „Funktionslogik“ gelten. Warum und inwiefern soziale Systemtheorie $(-n)$ hier die theoretische Fundierung bedeuten, wird allerdings kaum (ausreichend) thematisiert. Im Sinn Niklas Luhmanns werden Massenmedien bestimmt als der Gesellschaft ein Instrument zur Selbstbeobachtung zur Verfügung stellend, ,aus dem die gesellschaftlichen Teilsysteme Orientierung beziehen“.

Die AutorInnen beanspruchen Originalität insofern, da sie das theoretische Konzept „Störung“ als relativ neu beschreiben im Vergleich zu Krisen-Konzepten. Beide Phänomenbereiche werden hier verstanden - im Anschluss an Andreas Schwarz und Martin Löffelholz - als ,hochgradig ambivalente Situationen, die mit geringer Wahrscheinlichkeit eintreten und potenziell schwere Folgen nach sich ziehen“", bis hin zur Gefährdung des jeweiligen Systems (S. 12). Dem Band zufolge ist der Störungsbegriff gegenüber dem Krisenbegriff fruchtbarer, weil Störungen früher wahrnehmbar und punktuell einsetzten und - gleichsam im Aufgreifen des Nachrichtenfaktors „Abgrenzbarkeit“ im Unterschied zu eher komplexen und andauernden Krisen begründet Anlass lieferten für Unterbrechungen des (medialen) Alltages durch entsprechende Sondersendungen.

Originell auch das Herausarbeiten der (scheinbar paradoxen) übergreifenden These, dass solche Sondersendungen ,eine Veralltäglichung des Außeralltäglichen“ leisteten (S. 19). In der Analyse ästhetischer Aspekte der Sendungen sehen die Herausgebenden ,eine besonders innovative Dimension“ ihres Vorgehens (S. 32ff). 
Methodisch liegt dem Band eine ethnografisch eingebettete Medienanalyse zugrunde. Das Filmmaterial wurde dabei besonders per hermeneutischer Videoanalyse untersucht, zudem wurden Interviews geführt.

Wie ein Vorgriff auf die Corona-Krisenkommunikation (und damit auch als Verweis auf Kontinuitäten in dieser Hinsicht) kann gelesen werden, dass typischerweise in solchen unklaren Lagen die entscheidenden Personen ,nicht über ein Wissen verfügen, das sie zum zielgerichteten Handeln befähigt und die Konsequenzen des eigenen Vorgehens vollständig absehen" ließe (S. 16). Dass die Herausgebenden ähnlich wie auf theoretischer Ebene „,das System“, in praktischer Hinsicht den Staat und die Regierung, kaum in Frage stellen (mögen), zeigt ihre Behauptung, ,,wenn (staatliche Behörden) Krisen-Kommunikation betreiben, dann geht es in der Regel nicht um Verteidigungsstrategien für die Organisation, sondern um Schadensabwendung für die Bevölkerung“. Wird solch ein Postulat in die Corona-Zeit fortgeschrieben, kann sich die Fragwürdigkeit solcher Annahmen zeigen. Bemerkenswert, dass es reichen soll, verunsicherten Menschen „ein Gefühl“ von Sicherheit zu vermitteln (statt z. B. für Sicherheit zu sorgen).

Was etwaigen praktischen Nutzwert angeht, wird das Spektrum der Feldakteure wie folgt bestimmt und untersucht: RedakteurInnen, ModeratorInnen, politische AkteurInnen und ExpertInnen. Für sämtliche Akteursgruppierungen ergeben sich hier praktische Anregungen, insofern diese kommunikativen Rollen auch im Sinne Erving Goffmans konstruiert und dargestellt werden (müssen). Etwaige (Reste der) "Watchdog"-Motive finden sich eher bei Moderierenden als in redaktioneller Arbeit. Interessant mit Blick auf journalismus(selbst-)kritische Diskurse die Erkenntnisse über Einflüsse von Programmdirektoren, Intendanten und Gremien auf Ansetzung und Gestaltung der Sendungen. Kritische Ansätze (wie von Uwe Krüger zur relativ starken „Homogenität des deutschen Mainstream-Journalismus“ oder von Michael Haller zum nicht nur gelegentlich ,moralisierenden Belehrungsjournalismus“) werden zumindest in einigen Passagen der Studie aufgegriffen und darauf verwiesen, dass Vertrauen in etablierte Medien im gesamten globalen Norden abzunehmen scheint.

Was die ästhetischen Qualitäten angeht, gibt es gelegentlich Probleme mit Wortwiederholungen, Kommata und Kongruenz. Zudem verwundert es, dass der Band ,in traditioneller Weise (sic!) die maskuline Genus-Form “ benutzt, und dass damit laut Fußnote ,jeweils männliche und weibliche Akteure" bezeichnet werden, also offenbar in dieser Reihenfolge und diverse Identitäten vernachlässigend. Bemerkenswert bei einem Band, der sich „medialen Störungen“ widmet.

Nichtsdestotrotz enthält der Band in vielerlei Hinsicht Anregendes, für eher Theoretisches oder mehr Praktisches. Was die Herausgebenden den Sendungsteams attestieren, mag auch für die AutorInnen dieses Bandes gelten: Sie leisten wichtige Beiträge zur ,politischen Medienkultur in Deutschland“.

Funding Open Access funding enabled and organized by Projekt DEAL.

Open Access Dieser Artikel wird unter der Creative Commons Namensnennung 4.0 International Lizenz veröffentlicht, welche die Nutzung, Vervielfältigung, Bearbeitung, Verbreitung und Wiedergabe in jeglichem Medium und Format erlaubt, sofern Sie den/die ursprünglichen Autor(en) und die Quelle ordnungsgemäß nennen, einen Link zur Creative Commons Lizenz beifügen und angeben, ob Änderungen vorgenommen wurden. 
Die in diesem Artikel enthaltenen Bilder und sonstiges Drittmaterial unterliegen ebenfalls der genannten Creative Commons Lizenz, sofern sich aus der Abbildungslegende nichts anderes ergibt. Sofern das betreffende Material nicht unter der genannten Creative Commons Lizenz steht und die betreffende Handlung nicht nach gesetzlichen Vorschriften erlaubt ist, ist für die oben aufgeführten Weiterverwendungen des Materials die Einwilligung des jeweiligen Rechteinhabers einzuholen.

Weitere Details zur Lizenz entnehmen Sie bitte der Lizenzinformation auf http://creativecommons.org/ licenses/by/4.0/deed.de.

Prof. Dr. Sebastian Köhler ist Professor für Publikation und Journalismus an der HMKW - Hochschule für Medien, Kommunikation und Wirtschaft in Berlin sowie Publizist. 